\title{
Association of Type of Antidepressant Initiation with Bleeding Risk in Atrial Fibrillation Patients Taking Oral Anticoagulants
}

\author{
Iris Yuefan Shao ${ }^{1}$. $\cdot J^{\prime}$ Neka S. Claxton ${ }^{1} \cdot$ Pamela L. Lutsey ${ }^{2} \cdot$ Lin Yee Chen $^{3} \cdot$ Richard F. MacLehose $^{2} \cdot$ Alvaro Alonso $^{1}$
}

Accepted: 2 May 2021 / Published online: 20 May 2021

(c) The Author(s) 2021

\begin{abstract}
Background Inconsistent evidence suggests that use of certain antidepressants, particularly selective serotonin reuptake inhibitors (SSRIs), in patients using oral anticoagulants (OACs) might be associated with an elevated risk of bleeding.

Objective This study aims to investigate the risk of bleeding associated with initiation of different types of antidepressants among atrial fibrillation (AF) patients on OAC therapy.

Patients and methods A total of 30,336 AF patients (mean age 72.2 years; 54\% female) on OAC therapy that started antidepressant treatment were identified from the Truven Health Analytics MarketScan Commercial and Medicare Databases for the period 2007-2015. Exposure was defined as filling a prescription for antidepressant, and categorized as SSRI, serotonin/ norepinephrine reuptake inhibitors (SNRIs), serotonin reuptake inhibitors (SRIs), tricyclic antidepressants (TCAs), or other antidepressants. The primary outcome was incident hospitalized bleeding. Associations of antidepressant type with bleeding were assessed calculating hazard ratios (HRs) and 95\% confidence intervals (CIs) with adjusted Cox models in pairwise propensity score-matched cohorts.

Results During a mean follow-up of 21 months, we identified 1612 bleeding episodes. In pairwise comparisons, SSRI use was associated with an increased risk of bleeding when compared to most other antidepressants (HR 1.22, 95\% CI 0.96-1.54 vs SNRI; HR $1.10,95 \%$ CI $0.90-1.35$ vs SRI; HR 1.03 , 95\% CI $0.82-1.30$ vs TCA). SNRI use was associated with the lowest bleeding risk. Results did not differ by OAC type, age, and sex.

Conclusions Among AF patients on OAC initiating antidepressants, risk of bleeding varied across antidepressant type. This information can inform treatment choices among patients receiving OAC.
\end{abstract}

Results from this study were previously presented as a poster presentation at American Heart Association EPI Lifestyle Scientific Session, 2019. Research reported in this publication was supported by the National Institutes of Health under Award Numbers R01HL122200, R21AG058445, and K24HL14852. The content is solely the responsibility of the authors and does not necessarily represent the official views of the National Institutes of Health. This work was additionally supported by American Heart Association grant 16EIA26410001.

Iris Yuefan Shao

yshao45@emory.edu

1 Department of Epidemiology, Rollins School of Public Health, Emory University, 1518 Clifton Rd NE, Atlanta, GA 30322, USA

2 Division of Epidemiology and Community Health, School of Public Health, University of Minnesota, Minneapolis, MN, USA

3 Cariovascular Division, Department of Medicine, University of Minnesota Medical School, Minneapolis, MN, USA

\section{Key Points}

Among atrial fibrillation patients taking oral anticoagulants, initiation of selective serotonin reuptake inhibitors was associated with an increased risk of bleeding when compared to most other types of antidepressants.

Among atrial fibrillation patients taking oral anticoagulants, initiation of serotonin/norepinephrine reuptake inhibitor was associated with the lowest bleeding risk.

Associations observed in this study did not differ by sex, age, or the type of oral anticoagulant used. 


\section{Introduction}

Atrial fibrillation (AF) is a common arrhythmia more frequently diagnosed among older adults $[1,2]$. The use of oral anticoagulants (OACs) remains the most common management strategy among AF patients to reduce the risk of stroke and thromboembolism [3]. Direct oral anticoagulants (DOACs) are now increasingly prescribed in clinical practice because of their potential advantages over warfarin, a vitamin $\mathrm{K}$ antagonist, including better effectiveness for stroke and systemic embolism prevention, and fewer drug-drug interactions, especially among AF patients without moderate-to-severe mitral stenosis or a mechanical heart valve [4-8].

Prior studies have shown that warfarin is associated with an elevated risk of bleeding when used concurrently with medications such as selective serotonin reuptake inhibitors (SSRIs) as compared to being used alone, potentially due to SSRIs' interference with warfarin metabolism [9-11]. Antidepressants, including SSRIs, remain key in the clinical management of depression [12]. Evidence has suggested that use of specific antidepressants, mainly SSRIs, is associated with an increased risk of bleeding versus other antidepressants [13, 14] In addition, some studies have suggested that concurrent use of SSRI and DOACs is associated with an elevated risk of bleeding among nonvalvular AF patients $[15,16]$. However, no studies to date explored the risk of bleeding associated with initiation of different antidepressants among AF patients on DOACs. Using a large commercial healthcare claims database, we investigated the risk of bleeding associated with initiation of different types of antidepressant among AF patients on OAC therapy, and the potential interaction between type of antidepressant and OAC (warfarin vs DOACs). Given the higher risk of bleeding among warfarin users as compared to DOAC users, we hypothesized that SSRI initiation would increase the risk of bleeding among patients on OAC therapy compared to other antidepressant types. We also hypothesized that the association between SSRI use and bleeding would be stronger among warfarin users than DOAC users.

\section{Methods}

\subsection{Study Population}

This study used administrative claims data from the Truven Health MarketScan ${ }^{\circledR}$ Commercial Claims and Encounters Database and the Medicare Supplemental and Coordination of Benefits Database (Truven Health Analytics Inc., Ann Arbor, MI) [17]. The MarketScan Commercial database included claims data covering individuals enrolled in commercial health insurance plans in the United States. The Medicare Supplemental and Coordination of Benefits database provided additional data on individuals with Medicare supplemental plans. The databases provide comprehensive information on patients' enrollment data, inpatient and outpatient claims, and outpatient pharmaceutical dispensings. The institutional review board at Emory University reviewed and approved this study and waived the need for patient consent.

In this analysis, we included individuals enrolled in the MarketScan databases between 2007 and 2015. We selected individuals with a prior diagnosis of nonvalvular AF receiving OAC therapy (either warfarin or DOAC), initiating an antidepressant after receiving OAC therapy, and having at least 180 days of continuous enrollment without antidepressant prescription filling after OAC initiation. AF patients' OAC initiation was captured based on their first OAC prescription filling after AF diagnosis. The index date for our analysis was defined as the first antidepressant prescription filling date no less than 180 days after enrollment. AF history was defined as the presence of International Classification of Disease Ninth Revision Clinical Modification (ICD-9 CM) code 427.31 or 427.32 on any position in at least one inpatient claim or two consecutive outpatient claims, 7 days to 1 year apart, and without any inpatient history of mitral stenosis (ICD-9 CM code 394.0) or mitral valve disorders (ICD-9 CM code 424.0) [18]. This study was reviewed and approved by the Institutional Review Board at Emory University.

\subsection{Oral Anticoagulant and Antidepressant Use}

MarketScan outpatient pharmaceutical claims database included individual prescription filling date and the National Drug Code. Based on this information, all eligible study enrollees' OAC (warfarin, dabigatran, rivaroxaban, apixaban) prescriptions between 2007 and 2015 were identified. We excluded all edoxaban users since it was not approved on the market by the US Food and Drug Administration until 2015.

As with the selection of OAC prescriptions, we identified all eligible patients' antidepressant prescription information using the pharmaceutical claims data. For this analysis, we included all drugs coded under the category of antidepressants in the claims database. Antidepressants were categorized as SSRI, serotonin/norepinephrine reuptake inhibitors (SNRIs), serotonin reuptake inhibitors (SRIs), tricyclic antidepressants (TCAs), and other (tetracyclic antidepressant, alpha-2 antagonist, and dopamine/norepinephrine reuptake inhibitor). A list of generic drug names under each category is included in supplemental Table S1 (see the electronic supplementary material). 


\subsection{Bleeding Outcome Definition}

The primary outcome of interest was the first hospitalization for hemorrhage and bleeding after initiation of antidepressant among AF patients on oral anticoagulation. Bleeding was defined by the presence of ICD-9 CM diagnostic codes for hemorrhage including organ-specific hemorrhage and all-cause bleeding, using a previously published algorithm by Fang et al. [19].

\subsection{Covariates}

We selected the following covariates as potential confounders for the association between antidepressant initiation and risk of bleeding: sex, age at antidepressant initiation, comorbidities (heart failure, hypertension, diabetes mellitus, myocardial infarction, peripheral arterial diseases, kidney failure, stroke, bleeding, anemia, coagulopathy, cancer, mood disorders, cognitive impairment, liver disease, chronic obstructive pulmonary disease, alcohol use), other medication use prior to index date (antiplatelet, diuretics, digoxin, lipid-lowering medications, antiarrhythmics, beta-blockers, angiotensinconverting-enzyme inhibitors, angiotensin II receptor blockers, calcium channel blockers), $\mathrm{CHA}_{2} \mathrm{DS}_{2}$-VASc score, and HAS-BLED score [20]. All covariate definitions were created using inpatient, outpatient, and pharmaceutical claims data. Diagnostic codes used to define comorbidities are presented in supplemental Table S2 (see the electronic supplementary material).

\subsection{Statistical Analysis}

Primary analyses compared the risk of bleeding hospitalization across five categories of antidepressants (TCA [reference group], SSRI, SNRI, SRI, and other) among AF patients on OAC. Follow-up time started at the time of the initial antidepressant prescription and ended when the patient had a bleeding event or disenrolled from the database. Cox proportional hazard models using time to first bleeding hospitalization as the outcome variable were used to estimate hazard ratios (HRs) and $95 \%$ confidence intervals (CIs). Regression models adjusted for all potential confounders described previously. All analyses followed an intentionto-treat approach and, therefore, did not take into consideration switching or discontinuation of the initial antidepressant regime.

We conducted additional analyses performing pairwise comparisons of all combination of SSRI, SNRI, SRI, and TCA among eligible populations after propensity-score (PS) matching. We calculated PSs for initiating a certain type of antidepressant treatment for each comparison, using logistic regression models including all previously described covariates as predictors. For each comparison, eligible patients were matched 1:1 by treatment group with a caliper of 0.2 standard deviations of the PS using the gmatch macro [21]. We did not include the "other" antidepressant category in the pairwise analyses due to the small number of antidepressant users among each sub-category within that group. We ran Cox proportional hazard models to calculate HRs and 95\% CIs in PS-matched cohorts. In the full model, we adjusted for all covariates listed previously to address potential residual confounding after PS matching.

Finally, we evaluated whether associations of antidepressant type with bleeding differed by OAC treatment, age, and sex, performing stratified analyses and including multiplicative terms in the models to test for interactions. In addition, we assessed the presence of additive interaction by calculating the relative excess risk due to interaction (RERI) [22].

\section{Results}

Among 678,683 AF patients receiving OAC (edoxaban users excluded, $N=144$ ) in the MarketScan database, 30,336 initiated an antidepressant and had at least 180 days of enrollment after first OAC use. More than $50 \%$ of patients $(N=$ $16,830,56 \%)$ were SSRI users, followed by other antidepressants $(N=4026,13 \%)$, SRI $(N=3486,12 \%)$, SNRI $(N=$ $3023,10 \%)$, and TCA $(N=2971,10 \%)$. Baseline characteristics of patients at the time of antidepressant initiation did not differ appreciably by type of antidepressant. Patients that initiated SRI were slightly older and more likely to be female as compared to all other antidepressant initiators (Table 1). Mean $\mathrm{CHA}_{2} \mathrm{DS}_{2}$-VASc score and HAS-BLED score were comparable across all groups (Table 1 ).

After a mean follow-up of 21 months, 1612 bleeding episodes were identified, with the highest rate of bleeding hospitalizations observed among SSRI users (incidence rate $=$ 3.4 per 100 person-years) and the lowest among SNRI users (incidence rate $=2.6$ per 100 person-years) (Table 2). After adjusting for potential confounders, SSRI users had a small increased hazard of bleeding risk compared to TCA initiators $(\mathrm{HR}=1.17,95 \% \mathrm{CI} 0.99-1.39)$. There were no clear associations between initiation of other antidepressants (SRI, SNRI, and other, compared to TCA) and the risk of bleeding (Table 2). To further evaluate whether discontinuation of OAC therapy prior to index date would have affected our observed effect estimates, an additional analysis excluding those that did not fill a new OAC prescription in the 90 days prior to index date was performed. Results from the additional analysis did not meaningfully differ from observed associations (supplemental Table S3; see the electronic supplementary material).

Using PS matching, six additional pairwise PS-matched comparison cohorts were generated. Compared to all other types of antidepressants, SSRI users had a higher rate of 
Table 1 Baseline characteristics of AF patients on oral anticoagulation by types of antidepressant, MarketScan ${ }^{\circledR}$, 2007-2015

\begin{tabular}{|c|c|c|c|c|c|}
\hline & SSRI & SNRI & SRI & TCA & Other \\
\hline$N(\%)$ & $16830(56)$ & $3023(10)$ & $3486(12)$ & $2971(10)$ & $4026(13)$ \\
\hline \multicolumn{6}{|l|}{ Mean (SD) } \\
\hline Age & $71.8(13.2)$ & $70.9(12.3)$ & $73.6(12.6)$ & $70.8(12.4)$ & $74.5(13.4)$ \\
\hline HAS-BLED Score & $2.5(1.3)$ & $2.5(1.3)$ & $2.6(1.3)$ & $2.4(1.3)$ & $2.7(1.3)$ \\
\hline $\mathrm{CHA}_{2} \mathrm{DS}_{2}-\mathrm{VASc}$ & $4.3(2.1)$ & $4.2(2.1)$ & $4.3(2.0)$ & $4.0(2.0)$ & $4.5(2.1)$ \\
\hline \multicolumn{6}{|l|}{$N(\%)$} \\
\hline Female & $8838(53)$ & $1570(52)$ & 2077 (60) & $1643(55)$ & $2224(55)$ \\
\hline $\mathrm{HF}$ & $7584(45)$ & $1358(45)$ & $1700(49)$ & $1187(40)$ & $2050(51)$ \\
\hline Hypertension & $14220(84)$ & $2618(87)$ & $3041(87)$ & $2473(83)$ & $3431(85)$ \\
\hline $\mathrm{DM}$ & $6187(37)$ & $1339(44)$ & $1265(36)$ & 1104 (37) & $1425(35)$ \\
\hline MI & $2361(14)$ & $401(13)$ & $561(16)$ & $362(12)$ & $609(15)$ \\
\hline PAD & $3930(23)$ & $790(26)$ & $866(25)$ & $675(23)$ & $1124(28)$ \\
\hline Kidney failure & $2861(17)$ & $522(17)$ & $724(21)$ & $491(17)$ & $844(21)$ \\
\hline Stroke & $5597(33)$ & $921(30)$ & $1080(31)$ & $867(29)$ & $1426(35)$ \\
\hline Bleeding (all types) & $6332(38)$ & $1104(37)$ & $1300(37)$ & $1051(35)$ & $1643(41)$ \\
\hline Intracranial or GI bleeding & $301(2)$ & $49(2)$ & $58(1)$ & $50(1)$ & $60(1)$ \\
\hline Anemia & $5607(33)$ & $1105(37)$ & $1272(36)$ & $972(33)$ & $1576(39)$ \\
\hline Coagulopathy & $2346(14)$ & $440(15)$ & $472(14)$ & $436(15)$ & $615(15)$ \\
\hline Cancer & $3343(20)$ & $632(21)$ & $722(21)$ & $600(20)$ & $940(23)$ \\
\hline Mood disorder & $4592(27)$ & $782(26)$ & $478(14)$ & $266(9)$ & $1143(28)$ \\
\hline Cognitive impairment & $1675(10)$ & $234(8)$ & $356(10)$ & $153(5)$ & $526(13)$ \\
\hline COPD & $6360(38)$ & 1174 (39) & $1383(40)$ & $1117(38)$ & $1773(44)$ \\
\hline Liver & $1322(8)$ & $285(9)$ & $315(9)$ & $319(11)$ & $394(10)$ \\
\hline Alcohol & $485(3)$ & $80(3)$ & $148(4)$ & $78(3)$ & $161(4)$ \\
\hline Antiplatelet & $463(3)$ & $87(3)$ & $95(3)$ & $82(3)$ & $119(3)$ \\
\hline Diuretics & $9940(59)$ & $1854(61)$ & $2160(62)$ & $1739(59)$ & $2418(60)$ \\
\hline Antiarrhythmic & $2155(13)$ & $377(12)$ & $434(12)$ & $425(14)$ & $488(12)$ \\
\hline Digoxin & $4602(27)$ & $807(27)$ & $937(27)$ & $807(27)$ & $1212(30)$ \\
\hline ACEI & $7513(45)$ & $1284(42)$ & $1635(47)$ & $1286(43)$ & $1784(44)$ \\
\hline ARB & $4671(28)$ & $872(29)$ & 1018 (29) & $828(28)$ & $1052(26)$ \\
\hline Beta-blocker & $13265(79)$ & $2378(79)$ & $2823(81)$ & 2347 (79) & $3172(79)$ \\
\hline Calcium channel blocker & $8144(48)$ & $1423(47)$ & 1704 (49) & $1484(50)$ & $1913(48)$ \\
\hline Lipid-lowering medications & $10898(65)$ & $1964(65)$ & $2334(67)$ & $1923(65)$ & $2502(62)$ \\
\hline Warfarin & $13652(81)$ & $2369(78)$ & $2777(80)$ & $2482(84)$ & $3214(80)$ \\
\hline Apixaban & $467(3)$ & $89(3)$ & $107(3)$ & $63(2)$ & $126(3)$ \\
\hline Rivaroxaban & $1307(8)$ & $282(9)$ & $318(9)$ & $221(7)$ & $359(9)$ \\
\hline Dabigatran & $1403(8)$ & $282(9)$ & $284(8)$ & $205(7)$ & $327(8)$ \\
\hline
\end{tabular}

$A C E I$ angiotensin-converting-enzyme inhibitor, $A R B$ angiotensin receptor blockers, $C O P D$ chronic obstructive pulmonary disease, $D M$ diabetes mellitus, $G I$ gastrointestinal, $H F$ heart failure, $H T N$ hypertension, $M I$ myocardial infarction, Other other antidepressants, $P A D$ peripheral arterial diseases, SNRI serotonin/norepinephrine reuptake inhibitor, SRI serotonin reuptake inhibitor, SSRI selective serotonin reuptake inhibitor, $T C A$ tricyclic antidepressant bleeding, with the highest bleeding risk when compared to SNRI users (HR $=1.22,95 \%$ CI 0.96-1.54; Table 3). In contrast, patients that initiated SNRIs experienced the lowest hazard of bleeding across all comparisons, especially when compared to SRI users ( $\mathrm{HR}=0.87,95 \%$ CI 0.67-1.11; Table 3). Pairwise comparison of TCA initiators with SSRI, SNRI, and SRI initiators showed no differences in the rate of bleeding between these groups (Table 3).
The associations did not differ by type of OAC used or sex (Table 4). When evaluating the interaction on a multiplicative scale between type of antidepressants initiated and age, results remained the same across all groups except for the comparison of SSRI versus SRI. SSRI initiation was associated with an almost $30 \%$ higher hazard of bleeding compared to SRI initiation among patients $>75$ years old $(\mathrm{HR}=1.32$, 95\% CI 1.02-1.69; Table 4). However, patients 
Table 2 Risk of bleeding associated with type of antidepressant in atrial fibrillation patients taking oral anticoagulants, unmatched sample, MarketScan ${ }^{\circledR}, 2007-2015$

\begin{tabular}{llllll}
\hline & TCA & SSRI & SNRI & SRI & Other \\
\hline No. events & 156 & 958 & 126 & 170 & 202 \\
Person-years & 5562 & 27895 & 4812 & 5207 & 5852 \\
$\begin{array}{l}\text { Incidence rate per 100 } \\
\text { person-years (95\% CI) }\end{array}$ & $2.81(2.38-3.28)$ & $3.43(3.22-3.66)$ & $2.62(2.18-3.12)$ & $3.27(2.79-3.79)$ & $3.45(2.99-3.96)$ \\
$\begin{array}{l}\text { Hazard ratio (95\% CI) } \\
\quad \text { Model 1 }\end{array}$ & 1 & & & & $1.05(0.84-1.30)$ \\
$\quad$ Model 2 & 1 & $1.17(0.99-1.38)$ & $0.94(0.74-1.19)$ & $1.09(0.89-1.34)$ \\
\hline
\end{tabular}

CI confidence interval, Other other antidepressants, SNRI serotonin/norepinephrine reuptake inhibitor, SRI serotonin reuptake inhibitor, SSRI selective serotonin reuptake inhibitor, $T C A$ tricyclic antidepressant

${ }^{a}$ Model 1 was adjusted for age and sex

${ }^{\mathrm{b}}$ Model 2 was adjusted for age, sex, HAS-BLED score, $\mathrm{CHA}_{2} \mathrm{DS}_{2}$-VASc score, alcohol use, comorbid conditions (heart failure, hypertension, diabetes, myocardial infarction, peripheral artery disease, kidney failure, stroke, bleeding, anemia, coagulopathy, cancer, mood disorder, cognitive impairment, chronic obstructive pulmonary disease, and liver disease) and other medications (antiplatelet, diuretic drugs, antiarrhythmic drugs, digoxin, angiotensin-converting-enzyme inhibitors, angiotensin receptor blockers, beta-blockers, calcium channel blockers, and lipid-lowering medications

initiating SSRI $\leq 75$ years old had 0.79 times the rate of bleeding risk compared to those that initiated SRIs $(\mathrm{HR}=$ $0.79,95 \%$ CI $0.56-1.12$; $p$ for multiplicative interaction $=$ 0.02 ; Table 4). There was no statistically significant interaction between type of antidepressant initiated and sex or age on an additive scale across all comparison groups. Nonetheless, there was statistically significant sub-additive interaction between type of antidepressant initiated and type of OAC used when comparing SSRI initiators to TCA initiators $(\mathrm{RERI}=-0.69,95 \% \mathrm{CI}-1.23$ to -0.15 ; Table 4$)$. Patients on warfarin that initiated SSRI had 1.06 times the rate of bleeding risk compared to those that initiated TCAs $(\mathrm{HR}=$ 1.06, 95\% CI 0.85-1.35; Table 4). Meanwhile, patients on DOAC that initiated SSRI had 1.18 times the rate of bleeding risk compared to those that initiated TCA $(\mathrm{HR}=1.18$, 95\% CI 0.40-3.45; Table 4).

\section{Discussion}

Using a large healthcare claims database, our study showed that AF patients on OAC therapy that started SSRIs, compared to other antidepressants, had the highest hazard of bleeding. The rate of bleeding was 1.23 times higher among SSRI initiators when compared to those that initiated SNRIs. Patients that initiated SNRIs had the lowest hazard of bleeding among all antidepressants. Among AF patients $>75$ years old, but not among younger patients, initiation of SSRI (versus SRI) was associated with a higher rate of bleeding in the multiplicative scale. Comparing to SSRIs, TCAs were associated with lower increases in bleeding risk among warfarin users than DOAC users on an additive scale. We did not find evidence of other differences in the hazard of bleeding associated with antidepressant initiation by specific OAC type, sex, or age.

Both OAC and certain types of antidepressants are associated with an increased risk of bleeding [23-25]. Previous studies have reported the association of antidepressant use with bleeding risk in OAC users. SSRI use has been associated with an increased risk of bleeding among OAC users in some studies [26, 27], but not others [28-30]. The findings could potentially be explained by SSRIs' interference with hepatic cytochrome P-450 isoenzyme metabolic pathways, which are responsible for warfarin metabolism [31]. Commonly prescribed SSRIs, including sertraline, citalopram, paroxetine, fluvoxamine, and fluoxetine, all have mild to substantial effect on cytochrome P-450 isoenzymes, thus increasing prothrombin time and altering the effect of warfarin [32, 33]. In our study, SSRI initiation was associated with a higher rate of bleeding risk and such a finding did not differ by OAC type (warfarin vs DOAC) for most pairwise comparisons. SNRIs, a type of dual action antidepressant, are less commonly prescribed to patients for treatment of depression or anxiety disorder than SSRIs, potentially due to their side effects [34]. Nonetheless, existing evidence showed that SNRIs do not increase the risk of bleeding [24] and when used concurrently with warfarin, SNRIs have very low (or no) inhibition effect on cytochrome P-450 isoenzyme metabolism [27, 33]. Consistent with results from prior studies, in the present study AF patients that initiated SNRIs had a lower rate of bleeding than did initiators of other antidepressant therapies. TCAs are not commonly recommended for cardiac patients, especially given the controversial evidence on their effect on arrhythmia and electrocardiogram abnormalities $[35,36]$. In our study, only $9 \%$ of patients filled a prescription for a TCA. Similar to one 
Table 3 Risk of bleeding associated with type of antidepressant in atrial fibrillation patients taking oral anticoagulants, PS-matched cohorts, MarketScan ${ }^{\circledR}, 2007-2015$

\begin{tabular}{|c|c|c|}
\hline SSRI vs SNRI & SNRI & SSRI \\
\hline No. events & 126 & 161 \\
\hline Incidence rate $^{\mathrm{a}}$ & 2.62 & 3.18 \\
\hline Hazard ratio $(95 \% \mathrm{CI})^{\mathrm{b}}$ & 1 (ref.) & $1.22(0.96-1.54)$ \\
\hline SSRI vs SRI & SRI & SSRI \\
\hline No. events & 170 & 206 \\
\hline Incidence rate ${ }^{\mathrm{a}}$ & 3.27 & 3.50 \\
\hline Hazard ratio $(95 \% \mathrm{CI})^{\mathrm{b}}$ & 1 (ref.) & $1.10(0.90-1.35)$ \\
\hline SSRI vs TCA & TCA & SSRI \\
\hline No. events & 156 & 147 \\
\hline Incidence rate ${ }^{\mathrm{a}}$ & 2.81 & 2.85 \\
\hline Hazard ratio $(95 \% \mathrm{CI})^{\mathrm{b}}$ & 1 (ref.) & $1.03(0.82-1.30)$ \\
\hline SNRI vs SRI & SRI & SNRI \\
\hline No. events & 132 & 118 \\
\hline Incidence rate ${ }^{\mathrm{a}}$ & 3.09 & 2.62 \\
\hline Hazard ratio $(95 \% \mathrm{CI})^{\mathrm{b}}$ & 1 (ref.) & $0.87(0.67-1.11)$ \\
\hline SNRI vs TCA & TCA & SNRI \\
\hline No. events & 129 & 113 \\
\hline Incidence rate $^{\mathrm{a}}$ & 2.66 & 2.67 \\
\hline Hazard ratio $(95 \% \mathrm{CI})^{\mathrm{b}}$ & 1 (ref.) & $0.98(0.76-1.27)$ \\
\hline SRI vs TCA & TCA & SRI \\
\hline No. events & 137 & 155 \\
\hline Incidence rate $^{\mathrm{a}}$ & 3.01 & 2.81 \\
\hline Hazard ratio $(95 \% \mathrm{CI})^{\mathrm{b}}$ & 1 (ref.) & $0.98(0.77-1.23)$ \\
\hline
\end{tabular}

$C I$ confidence interval, $P S$ propensity score, SNRI serotonin/norepinephrine reuptake inhibitor, SRI serotonin reuptake inhibitor, SSRI selective serotonin reuptake inhibitor, TCA tricyclic antidepressant

${ }^{a}$ Incidence rate in events per 100 person-years

${ }^{b}$ Fully adjusted model corresponds to pairwise PS-matched cohorts. Models were adjusted for age, sex, HAS-BLED score, $\mathrm{CHA}_{2} \mathrm{DS}_{2}$-VASc score, alcohol use, comorbid conditions (heart failure, hypertension, diabetes, myocardial infarction, peripheral artery disease, kidney failure, stroke, bleeding, anemia, coagulopathy, cancer, mood disorder, cognitive impairment, chronic obstructive pulmonary disease, and liver disease) and other medications (antiplatelet, diuretic drugs, antiarrhythmic drugs, digoxin, angiotensin-converting-enzyme inhibitors, angiotensin receptor blockers, beta blockers, calcium channel blockers, and lipid-lowering medications)

previous study, we did not find evidence for increased hazard of bleeding hospitalization associated with initiation of TCA among patients on OAC [27].

Approximately one fifth of eligible study participants included in our analysis were DOAC users. Since few published studies have assessed the risk of bleeding associated with concomitant use of antidepressant and DOAC [28, 30], we performed additional stratified analyses and tested for interaction between antidepressant type and OAC type. Results from prior studies showed that SSRI use is not associated with an increased risk of bleeding among AF patients on DOAC [28, 30]. In our study, we did not find any evidence supporting a differential effect of antidepressant type on bleeding by type of OAC, other than a potential weaker association of SSRI versus TCA with bleeding risk in warfarin users than in DOAC users. This finding was unexpected and requires replication.

When stratifying by age, our study showed that the greater hazard of bleeding associated with SSRI initiation as compared to SRI initiation was restricted to AF patients > 75 years old. Nevertheless, the observed association between antidepressant initiation and bleeding hospitalization did not differ by age in any of the other comparison groups. Prior studies have suggested bleeding as a complication for elderly patients on either warfarin or antidepressants, but none have assessed whether such a finding holds for elderly patients taking warfarin and SSRIs concomitantly [37, 38]. Our observation could possibly be explained by unmeasured 
Table 4. Risk of bleeding associated with type of antidepressant in atrial fibrillation patients taking oral anticoagulants in PS-matched cohorts, stratified by sex, age, and oral anticoagulation type, MarketScan ${ }^{\circledR}, 2007-2015$

\begin{tabular}{|c|c|c|c|}
\hline & \multicolumn{3}{|c|}{ Hazard ratio $(95 \% \text { confidence interval })^{\mathrm{a}}$} \\
\hline & SSRI vs SNRI & SSRI vs SRI & SSRI vs TCA \\
\hline Warfarin & $1.23(0.96$ to 1.59$)$ & $1.11(0.89$ to 1.37$)$ & $1.06(0.85$ to 1.35$)$ \\
\hline DOAC & $1.10(0.56$ to 2.13$)$ & $1.03(0.54$ to 2.00$)$ & $1.18(0.40$ to 3.45$)$ \\
\hline P for multiplicative interaction & 0.92 & 0.86 & 0.42 \\
\hline RERI (95\% confidence interval) & $-0.07(-0.81$ to 0.67$)$ & $-0.06(-0.61$ to 0.49$)$ & $-0.69(-1.23$ to -0.15$)$ \\
\hline$P$ for additive interaction & 0.85 & 0.83 & 0.01 \\
\hline Male & $1.39(0.99$ to 1.39$)$ & $1.09(0.83$ to 1.43$)$ & $1.14(0.83$ to 1.56$)$ \\
\hline Female & $1.08(0.77$ to 1.49$)$ & $1.10(0.80$ to 1.49$)$ & $0.96(0.69$ to 1.33$)$ \\
\hline P for multiplicative interaction & 0.31 & 0.94 & 0.45 \\
\hline RERI (95\% confidence interval) & $0.24(-0.27$ to 0.74$)$ & $0.02(-0.37$ to 0.41$)$ & $0.15(-0.40$ to 0.70$)$ \\
\hline$P$ for additive interaction & 0.36 & 0.92 & 0.60 \\
\hline Age $>75$ years & $1.30(0.95$ to 1.79$)$ & $1.32(1.02$ to 1.69$)$ & $1.20(0.91$ to 1.59$)$ \\
\hline Age $\leq 75$ years & $1.14(0.79$ to 1.61$)$ & $0.79(0.56$ to 1.12$)$ & $0.81(0.55$ to 1.18$)$ \\
\hline$P$ for multiplicative interaction & 0.66 & 0.02 & 0.10 \\
\hline RERI ( $95 \%$ confidence interval) & $0.16(-0.28$ to 0.60$)$ & $0.44(-0.20$ to 1.08$)$ & $0.37(-0.23$ to 0.97$)$ \\
\hline \multirow[t]{2}{*}{$P$ for additive interaction } & 0.47 & 0.18 & 0.22 \\
\hline & SNRI vs TCA & SNRI vs SRI & SRI vs TCA \\
\hline Warfarin & $0.95(0.72$ to 1.23$)$ & $0.85(0.65$ to 1.11$)$ & $1.00(0.79$ to 1.28$)$ \\
\hline DOAC & $1.85(0.72$ to 4.76$)$ & $1.12(0.54$ to 2.32$)$ & $1.28(0.56$ to 3.03$)$ \\
\hline$P$ for multiplicative interaction & 0.37 & 0.55 & 0.43 \\
\hline RERI (95\% confidence interval) & $0.48(-0.30$ to 1.25$)$ & $0.21(-0.46$ to 0.88$)$ & $0.42(-0.22$ to 1.09$)$ \\
\hline$P$ for additive interaction & 0.23 & 0.54 & 0.22 \\
\hline Male & $1.08(0.74$ to 1.56$)$ & $0.84(0.58$ to 1.20$)$ & $1.16(0.84$ to 1.61$)$ \\
\hline Female & $0.94(0.65$ to 1.30$)$ & $0.88(0.62$ to 1.27$)$ & $0.93(0.66$ to 1.30$)$ \\
\hline P for multiplicative interaction & 0.51 & 0.68 & 0.41 \\
\hline RERI (95\% Confidence Interval) & $0.16(-0.39$ to 0.72$)$ & $-0.08(-0.56$ to 0.39$)$ & $0.20(-0.28$ to 0.69$)$ \\
\hline$p$ for additive interaction & 0.57 & 0.73 & 0.41 \\
\hline Age $>75$ years & $0.96(0.68$ to 1.35$)$ & $1.00(0.71$ to 1.41$)$ & $0.96(0.70$ to 1.30$)$ \\
\hline Age $\leq 75$ years & $1.01(0.69$ to 1.49$)$ & $0.70(0.48$ to 1.01$)$ & $1.19(0.83$ to 1.69$)$ \\
\hline P for multiplicative interaction & 0.72 & 0.16 & 0.35 \\
\hline RERI (95\% confidence interval) & $-0.12(-0.56$ to 0.33$)$ & $0.30(-0.03$ to 0.64$)$ & $-0.26(-0.63$ to 0.12$)$ \\
\hline$P$ for additive interaction & 0.61 & 0.07 & 0.18 \\
\hline
\end{tabular}

$D O A C$ direct oral anticoagulant, $P S$ propensity score, RERI relative excess risk due to Interaction, SNRI serotonin/norepinephrine reuptake inhibitor, SRI serotonin reuptake inhibitor, SSRI selective serotonin reuptake inhibitor, TCA tricyclic antidepressant

${ }^{a}$ All models corresponded to pairwise PS-matched cohorts. Models were adjusted for age, sex, HAS-BLED score, $\mathrm{CHA}_{2} \mathrm{DS}_{2}$-VASc score, alcohol use, comorbid conditions (heart failure, hypertension, diabetes, myocardial infarction, peripheral artery disease, kidney failure, stroke, bleeding, anemia, coagulopathy, cancer, mood disorder, cognitive impairment, chronic obstructive pulmonary disease, and liver disease) and other medications (antiplatelet, diuretic drugs, antiarrhythmic drugs, digoxin, angiotensin-converting-enzyme inhibitors, angiotensin receptor blockers, beta-blockers, calcium channel blockers, and lipid-lowering medications)

factors such as frailty and increased risk of bleeding at baseline for antidepressant users $>75$ years old. Due to limited sample size in each age-specific stratum, our findings need to be confirmed in future larger studies.

Our study has some strengths. This study has a large sample size and a sizable number of bleeding events, allowing for consideration of different types of antidepressants and OACs (warfarin vs DOACs). However, there are several limitations to this study design. First of all, due to the nature of a claims database, we obtained exposure, outcome, and covariate information using ICD-9 CM codebased definitions. The sensitivity, specificity, and positive predictive values of these definitions varies across conditions [39-42] Therefore, misclassification of exposure, outcome, and potential confounders could all contribute to biased estimates. Second, even though we tried to adjust 
for confounding using PS matching, residual confounding and confounding by indication might be present. TCAs, for example, have been reported to cause electrocardiogram changes [36]. AF patients, therefore, might be less likely to be prescribed with TCA to avoid potential cardiac complications and future risk of bleeding. Therefore, our estimates for the hazard of bleeding associated with TCA initiation might be biased since fewer AF patients at an elevated risk of bleeding would have been prescribed with TCA. Third, we cannot capture patients' precise OAC and antidepressant adherence behavior in the claims dataset. Certain psychiatric conditions such as major depressive disorders can lead to patients' lack of motivation [43]. Therefore, patients included in our study might be more prone to non-adherent behavior thus leading to biased estimate of bleeding risk associated with concomitant use of antidepressants and OAC. Lastly, a small number of bleeding hospitalization events in each stratum, especially for non-SSRI users, might hamper the ability to estimate precise associations. Given these limitations, estimates of effect from our study should be interpreted with caution.

\section{Conclusion}

To conclude, in AF patients on OAC therapy, initiation of SSRIs, as compared to all other types of antidepressants, is associated with an increased risk of bleeding, whereas SNRI initiation is associated with the lowest risk of bleeding among all antidepressant types. Results from this study could potentially assist with clinician and AF patients' decisions on choice of antidepressant when patients are concurrently taking OACs. Future studies are needed to further confirm the observed findings.

Supplementary Information The online version contains supplementary material available at https://doi.org/10.1007/s40801-021-00258-3.

\section{Declarations}

Funding Research reported in this publication was supported by the National Institutes of Health under Award Numbers R01HL122200, R21AG058445, and K24HL14852. The content is solely the responsibility of the authors and does not necessarily represent the official views of the National Institutes of Health. This work was additionally supported by American Heart Association grant 16EIA26410001 (Alonso).

Conflict of interest The authors declare that they have no competing interests.

Ethics approval The institutional review board at Emory University reviewed and approved this study and waived the need for patient consent.

Consent to participate Not applicable.
Consent for publication Not applicable.

Availability of data and material Because of licensing restrictions, data and study materials cannot be made available to other investigators to reproduce results, but researchers may contact IBM Watson Health to obtain and license the data.

Code availability Not applicable.

Author contributions All authors in this paper have contributed significantly to the design of the study, analysis and interpretation of results, and have participated in the drafting of the manuscript or have revised it critically for important intellectual content. Finally, all coauthors have seen and agree with the contents of the manuscript, and all authors believe that the manuscript represents honest work.

Open Access This article is licensed under a Creative Commons Attribution-NonCommercial 4.0 International License, which permits any non-commercial use, sharing, adaptation, distribution and reproduction in any medium or format, as long as you give appropriate credit to the original author(s) and the source, provide a link to the Creative Commons licence, and indicate if changes were made. The images or other third party material in this article are included in the article's Creative Commons licence, unless indicated otherwise in a credit line to the material. If material is not included in the article's Creative Commons licence and your intended use is not permitted by statutory regulation or exceeds the permitted use, you will need to obtain permission directly from the copyright holder. To view a copy of this licence, visit http://creativecommons.org/licenses/by-nc/4.0/.

\section{References}

1. Staerk L, Sherer JA, Ko D, Benjamin EJ, Helm RH. Atrial fibrillation: epidemiology, pathophysiology, clinical outcomes. Circ Res. 2017;120(9):1501-17.

2. Morillo CA, Banerjee A, Perel P, Wood D, Jouven X. Atrial fibrillation: the current epidemic. J Geriatr Cardiol. 2017;14(3):195-203.

3. Hsu J, Scheinman MM. Atrial fibrillation. In: Crawford MH, editors. Current diagnosis \& treatment: cardiology. 5th edn., chap. 12. New York: McGraw-Hill Education.

4. Vinogradova Y, Coupland C, Hill T, Hippisley-Cox J. Risks and benefits of direct oral anticoagulants versus warfarin in a real world setting: cohort study in primary care. BMJ. 2018:362:k2505.

5. Li G, et al. Direct comparative effectiveness and safety between non-vitamin $\mathrm{K}$ antagonist oral anticoagulants for stroke prevention in nonvalvular atrial fibrillation: a systematic review and meta-analysis of observational studies. Eur J Epidemiol. 2019;34(2):173-90.

6. Yao X, et al. Effectiveness and safety of dabigatran, rivaroxaban, and apixaban versus warfarin in nonvalvular atrial fibrillation. $\mathbf{J}$ Am Heart Assoc. 2016;5(6):1-18.

7. Graham DJ, Baro E, Zhang R, Liao J, Wernecke M. Comparative stroke, bleeding, and mortality risks in older medicare patients treated with oral anticoagulants for nonvalvular atrial fibrillation. Am J Med. 2019;132(5):596-604.e11.

8. January C, et al. 2019 AHA/ACC/HRS focused update of the 2014 AHA/ACC/HRS guideline for the management of patients with atrial fibrillation. J Am Coll Cardiol. 2019;74(1):104-32.

9. Quinn GR, et al. Selective serotonin reuptake inhibitors and bleeding risk in anticoagulated patients with atrial fibrillation: ananalysis from the ROCKET AF trial. J Am Heart Assoc. 2018;7:1-9. 
10. Schalekamp T, Klungel OH, Souverein PC, De Boer A. Increased bleeding risk with concurrent use of selective serotonin reuptake inhibitors and coumarins. Arch Intern Med. 2008;168(2):180-5.

11. Quinn GR, et al. Effect of selective serotonin reuptake inhibitors on bleeding risk in patients with atrial fibrillation taking warfarin. Am J Cardiol. 2014;114(4):583-6.

12. Victoria A-K, et al. Antidepressant prescribing in five European countries: application of common definitions to assess the prevalence, clinical observations, and methodological implications. Eur J Clin Pharmacol. 2014;70(7):849-57.

13. Paton C, Ferrier IN. SSRIs and gastrointestinal bleeding. BMJ. 2005;331(7516):529-30.

14. Renoux C, Vahey S, Dell'Aniello S, Boivin JF. Association of selective serotonin reuptake inhibitors with the risk for spontaneous intracranial hemorrhage. JAMA Neurol. 2017;74(2):173-80.

15. Zhang Y, Souverein PC, Gardarsdottir H, van den Ham HA, Maitland-van der Zee AH, de Boer A. Risk of major bleeding among users of direct oral anticoagulants combined with interacting drugs: a population-based nested case-control study. Br J Clin Pharmacol. 2020;86(6):1150-64.

16. Ibáñez L, et al. Incidence of direct oral anticoagulant use in patients with nonvalvular atrial fibrillation and characteristics of users in 6 European countries (2008-2015): a cross-national drug utilization study. Br J Clin Pharmacol. 2019;85(11):2524-39.

17. Hansen L. The Truven Health MarketScan Databases for life sciences researchers. IBM Watson Health. 2017.

18. Alonso A, et al. Prospective study of oral anticoagulants and risk of liver injury in patients with atrial fibrillation. Heart. 2017;103(11):834-9.

19. Fang MC, et al. A new risk scheme to predict warfarin-associated hemorrhage: the ATRIA (Anticoagulation and Risk Factors in Atrial Fibrillation) Study. J Am Coll Cardiol. 2011;58(4):395-401.

20. Lane DA, et al. Use of the CHA2DS2-VASc and HAS-BLED scores to aid decision making for thromboprophylaxis innonvalvular atrial fibrillation. Circulation. 2012;126(7):860-5.

21. Stuart EA. Matching methods for causal inference: a review and a look forward. Stat Sci. 2010;25(1):1-21.

22. Li R, Chambless L. Test for additive interaction in proportional hazards models. Ann Epidemiol. 2007;17(3):227-36.

23. Paton C, Ferrier IN. SSRIs and gastrointestinal bleeding. Br Med J. 2005;331(7516):529-30.

24. Cheng YL, et al. Use of SSRI, but not SNRI, increased upper and lower gastrointestinal bleeding a nationwide populationbased cohort study in Taiwan. Medicine (United States). 2015;94(46):e2022.

25. Bixby AL, VandenBerg A, Bostwick JR. Clinical management of bleeding risk with antidepressants. Ann Pharmacother. 2019;53(2):186-94

26. Cochran KA, Cavallari LH, Shapiro NL, Bishop JR. Bleeding incidence with concomitant use of antidepressants and warfarin. Ther Drug Monit. 2011;33(4):433-8.

27. Schelleman H, Brensinger CM, Bilker WB, Hennessy S. Antidepressant-warfarin interaction and associated gastrointestinal bleeding risk in a case-control study. PLoS ONE. 2011;6(6):1-6.
28. Quinn GR, et al. Selective serotonin reuptake inhibitors and bleeding risk in anticoagulated patients with atrial fibrillation: an analysis from the ROCKET AF trial. J Am Heart Assoc. 2018;7(15):1-9.

29. Spina E, Barbieri MA, Cicala G, Bruno A, de Leon J. Clinically relevant drug interactions between newer antidepressants and oral anticoagulants. Expert Opin Drug Metab Toxicol. 2020;16(1):31-44.

30. Sheikh Rezaei S, Mittlböck M, Reichardt B, Wolzt M. SSRI comedication with NOAC or VKA does not increase hospitalisation for bleeding: a retrospective nationwide cohort study in Austria 2010-2015. Int J Geriatr Psychiatry. 2019;34(8):1194-9.

31. Lynch T, Price A. The effect of cytochrome P450 metabolism on drug response, interactions, and adverse effects. Am Fam Physician. 2007;76(3):391-6.

32. Sciences RB, Teles JS, Fukuda EY, Feder D. Warfarin: pharmacological profile and drug interactions with antidepressants. Einstein (São Paulo, Brazil). 2012;10(11):110-5.

33. Sansone RA, Sansone LA. Warfarin and antidepressants: happiness without hemorrhaging. Psychiatry (Edgmont). 2009;6(7):24-9.

34. Santarsieri D, Schwartz TL. Antidepressant efficacy and sideeffect burden: a quick guide for clinicians. Drugs Context. 2015;4:212290.

35. Glassman AH, Roose SP, Bigger JT Jr. The safety of tricyclic antidepressants in cardiac patients: risk-benefit reconsidered. JAMA. 1993;269(20):2673-5.

36. Yekehtaz H, Farokhnia M, Akhondzadeh S. Cardiovascular considerations in antidepressant therapy: an evidence-based review. J Tehran Heart Cent. 2013;8(4):169-76.

37. Coupland C, Dhiman P, Morriss R, Arthur A, Barton G, Hippisley-Cox J. Antidepressant use and risk of adverse outcomes in older people: population based cohort study. BMJ. 2011;343:4551.

38. Darnell SW, Davis SC, Whitcomb JJ, Manfredi JA, McLaurin BT. Bleeding risk factors affecting warfarin therapy in the elderly with atrial fibrillation. Dimens Crit Care Nurs. 2014;33(2):57-63.

39. Thigpen JL, et al. Validity of international classification of disease codes to identify ischemic stroke and intracranial hemorrhage among individuals with associated diagnosis of atrial fibrillation. Circ Cardiovasc Qual Outcomes. 2015;8(1):8-14.

40. Jensen PN, Johnson K, Floyd J, Heckbert SR, Carnahan R, Dublin $\mathrm{S}$. A systematic review of validated methods for identifying atrial fibrillation using administrative data. Pharmacoepidemiol Drug Saf. 2012;21:141-7.

41. Quan H, et al. Assessing validity of ICD-9-CM and ICD-10 administrative data in recording clinical conditions in a unique dually coded database. Health Serv Res. 2008;43(4):1424-41.

42. Chen N, et al. Association of oral anticoagulant type with risk of dementia among patients with nonvalvular atrial fibrillation. J Am Heart Assoc. 2018;7(21).

43. Balán IC, Moyers TB, Lewis-Fernández R. Motivational pharmacotherapy: combining motivational interviewing and antidepressant therapy to improve treatment adherence. Psychiatry Interpers Biol Process. 2013;76(3):203-9. 